\title{
Discapacidad y Determinantes Sociales de la Salud Estructurales e Intermedios: Diferencias por Género
}

\section{Disability and Social Determinants of Structural Health and Intermediates: Differences by Gender}

Recibido: 14 Mayo 2014/Enviado para modificación: 2 de Julio 2014/Aceptado: 17 de Julio 2014

\author{
Consuelo Vélez Álvarez ${ }^{1}$ \\ Universidad Autónoma de Manizales \\ José Armando Vidarte Claros ${ }^{2}$ \\ Universidad Autónoma de Manizales
}

\section{RESUMEN}

Objetivo: Establecer los determinantes sociales de la salud estructurales e intermedios y su relación con la discapacidad en la ciudad de Barranquilla, a partir del análisis de las diferencias por género. Materiales y métodos: Estudio descriptivo correlacional, con 726 registros de la base de datos a 2011. Se utilizó el Registro DANE de personas con discapacidad, que fue sistematizado en el programa SPSS Versión 19.0. Resultados: Se encontraron diferencias estadísticamente significativas $(\mathrm{p}<0,05)$ y niveles de dependencia baja relacionadas con tipo de afiliación a la seguridad social, la raza, el trabajo desempeñado y el salario mensual. Conclusiones: Existen diferencias por género en algunos determinantes sociales de la salud. Además, la discapacidad se hace evidente cuando la persona encuentra o presenta restricciones que le impiden su plena participación en la sociedad.

Palabras Clave:Personas con discapacidad, condiciones sociales, limitación, deficiencia, participación social (Fuente: DeCS).

\section{ABSTRACT}

Objective:To establish the social determinants of health and intermediate structural and Disability in the city of Barranquilla, analyzing gender differences. Materials and Methods: A descriptive correlational study with 726 records database to 2011, the Registry was used DANE people with disabilities was systematized in the SPSS version 19.0 program. Results: Statistically significant differences $p<0.05$ and low levels of dependence affiliation to social security, race, work performed and met monthly salary. Conclusions: There are gender differences in some social Determinants of Health, disability is also evident when the person is or has restrictions that prevent their full participation in society.

Keywords:People with disabilities, social conditions, limitation, impairment, social participation(Source: MeSH, NLM).

Para citar este artículo: Velez C, VidarteJA. Discapacidad y Determinantes Sociales de la Salud Estructurales e Intermedios: Diferencias por Género. Cienc. innov. salud. 2014; 2 (2):63 - 69

\footnotetext{
${ }^{1}$ Enfermera. Especialista en Epidemiología, PhD. en Salud Pública. Docente Titular Universidad Autónoma de Manizales. Correo electrónico: cva@autonoma.edu.co

${ }^{2}$ Licenciado en Educación física. PhD. en Ciencias de la Actividad y el Deporte. Docente Titular Universidad Autónoma de Manizales. Correo electrónico: jovida@autonoma.edu.co.
} 


\section{Introducción}

Se entiende como determinantes sociales a las condiciones en que las personas nacen, crecen, viven, trabajan y envejecen; de modo que las desigualdades que se manifiestan en estas condiciones también crean desigualdades en salud (1). Por esta razón, hablar de determinantes sociales de la salud implica asumir una estrategia para aplicar el conocimiento científico acumulado a las causas últimas o estructuras de los problemas de salud, con el fin de establecer estrategias saludables para todos. Con ello se busca reconocer los elementos de la estructura social que condicionan la posición de las personas en la sociedad, y que afectan de manera directa la distribución de los problemas o los factores protectores de la salud (2).

El modelo de Dahlgren y Whitehead, adoptado por Acheson (3), presenta los principales determinantes de la salud como capas de influencia. En su centro, se encuentra el individuo y los factores constitucionales que afectan su salud y que son inmodificables; mientras que a su alrededor aparecen las capas que se refieren a determinantes posibles de cambiar, siendo los primeros los estilos de vida individuales, que, desde hace décadas, han sido el objeto de la promoción en salud. Luego se encuentra la capa en que los individuos son influenciados por su comunidad y las redes sociales o redes emocionales y familiares, las cuales pueden sostener la salud de los individuos o dificultar la adopción de conductas saludables cuando son ignoradas.

Los determinantes más amplios o profundos, en cuanto a su influencia, se relacionan con las condiciones de vida y trabajo, alimentos y acceso a servicios básicos, además de las condiciones socioeconómicas, culturales y ambientales, representadas en la capa más externa. El modelo descrito intenta representar, además, las interacciones entre estos determinantes.

Acorde con lo establecido en él, se puede plantear que los determinantes sociales en salud se clasifican en determinantes estructurales $\mathrm{e}$ intermedios. Los estructurales son aquellos que generan estratificación social, e incluyen factores tradicionales como los ingresos y la educación. En la actualidad, sin embargo, resulta también importante reconocer el género, la raza y la sexualidad como estratificadores sociales (4).
En esta perspectiva, la comisión sobre determinantes sociales en salud plantea que la estratificación por grupos sociales, educación, ocupación, género y otros factores, lleva a la aparición de inequidades sociales en condiciones de vida y de trabajo. Así, los mecanismos de estratificación socioeconómica, considerados determinantes estructurales de las inequidades en salud, configuran las oportunidades para su logro, según diferencias en vulnerabilidades, exposiciones a agentes y acceso a servicios básicos. Esto significa que la influencia de la posición socioeconómica del individuo sobre su salud no es directa, sino producto de factores intermediarios: condiciones materiales, tales como la calidad de vivienda, y circunstancias psicosociales que incluyen el estrés y comportamientos como el hábito de fumar o de alimentarse mal (3).

En este marco, según la perspectiva canadiense, la influencia de los determinantes sociales de la salud sobre la discapacidad, se explica por los siguientes factores: el ingreso y nivel social; las redes de apoyo social; la educación; el empleo y la condición de trabajo; los entornos sociales; los entornos físicos; las prácticas de salud personales y las aptitudes de adaptación; las características biológicas y genéticas; los servicios de salud; el género y la cultura (5).

Vale decir que la discapacidad es un concepto genérico que incluye déficits en funciones y estructuras, limitaciones en la actividad y restricciones en la participación, indicando con ello los aspectos negativos de la interacción entre un individuo (con una "condición de salud") y sus factores contextuales (factores ambientales y personales). La Clasificación Internacional del Funcionamiento, de la Discapacidad $y$ de la Salud (CIF), basada en un modelo biopsicosocial para abordar la salud y la discapacidad, entiende la discapacidad como "un fenómeno multidimensional resultado de la interacción entre la persona y su contexto físico y social"(6). Esta misma entidad designa el asunto tratado con la expresión Situación de Discapacidad, pues lo considera un problema creciente, y sitúa a todas las enfermedades y los problemas de salud en pie de igualdad independientemente de su causa.

La CIF proporciona una descripción de situaciones relacionadas con el funcionamiento humano y sus variaciones, así como la relación del contexto, brindando un lenguaje unificado, estandarizado, y un 
marco conceptual para la descripción de los componentes de la salud (6).

El análisis del funcionamiento y la discapacidad supone el abordaje de lo corporal: funciones, estructuras corporales y deficiencias en estructuras y funciones corporales, tanto en lo individual como en lo social: Es decir, actividades y participación, limitación en las actividades y restricción de la participación en la sociedad. Las funciones corporales comprenden las funciones fisiológicas de los sistemas corporales, incluyendo las funciones psicológicas; y las estructuras corporales se refieren a las partes anatómicas o estructuras del cuerpo como órganos y miembros. Estas proporcionan información sobre el estado funcional del individuo en lo referente a las partes anatómicas y fisiológicas del cuerpo, de allí su nombre.

Las deficiencias comportan problemas en las funciones o estructuras corporales, tales como una desviación significativa o una pérdida. Pueden ser temporales o permanentes, progresivas, regresivas o estáticas, intermitentes o continuas. Por otro lado, las actividades y la participación son los aspectos relacionados con el funcionamiento humano desde una perspectiva individual y social.

Por actividad se entiende la realización de una tarea o acción por una persona. Implica una perspectiva individual en un contexto normalizado; en tanto que la participación es el acto de involucrarse en una situación vital, e incluye una perspectiva social en un contexto real. Las limitaciones en la actividad definen las dificultades que una persona puede tener en el desempeño de las actividades; y las restricciones en la participación constituyen problemas que alguien puede experimentar al involucrarse en situaciones vitales.

Por su parte, los factores contextuales constituyen, en conjunto el contexto completo de la vida de un individuo. Se clasifican en factores ambientales y factores personales. Los ambientales se refieren a todos los aspectos del mundo extrínseco o externo que forman el contexto de la vida de un individuo, y como tal afecta su funcionamiento. Los factores personales constituyen el trasfondo particular de la vida de un individuo y su estilo de vida. Están compuestos por características del individuo que no forman parte de su condición o estado de salud. Estos pueden incluir el género, la raza, la edad, la forma física, los estilos de vida, los hábitos, la educación, la profesión, experiencias actuales y pasadas, el comportamiento, la personalidad y la espiritualidad, entre otros (6).

En Colombia, el recurso más significativo respecto a la información sobre discapacidad es el registro para la localización y caracterización de personas con discapacidad (DANE, 2003) (7). A partir de este registro, se han realizado procesos investigativos que intentan abordar este problema (8-12), pero no han conseguido un análisis integral de la situación desde la perspectiva de los determinantes sociales de la salud en esta población.

Algunos estudios sobre determinantes sociales y discapacidad han contribuido, no obstante, a comprender la relación entre la discapacidad y los diferentes ámbitos socioeconómicos, que podrían estar asociados a la prevalencia de las condiciones de discapacidad (13-15). De igual forma, se ha planteado que la discapacidad surge realmente cuando la persona que la padece se enfrenta a las condiciones sociales o del medio ambiente, estableciéndose, en consecuencia, que esta es una resultante de la operación de desactivación y discriminación desde las condiciones culturales, sociales y ambientales (15).

Acorde con todo lo propuesto, el objetivo de este trabajo consistió en establecer la relación entre la discapacidad y los determinantes sociales de la salud estructurales e intermedios en la ciudad de Barranquilla, analizando en concreto las diferencias por género. Con respesto a este último concepto, se asume el género como el conjunto de los roles socialmente construidos, los comportamientos, actividades y atributos que una sociedad dada considera apropiados para los hombres y las mujeres. Así, masculino y femenino son categorías de género (16).

\section{Materiales y Métodos}

El estudio se inscribe en la perspectiva cuantitativa, desde un análisis descriptivo con una fase correlacional. En su desarrollo, se analizaron 726 registros de personas con discapacidad de la ciudad de Barranquilla, identificadas en el registro de caracterización y localización de personas con discapacidad, según datos recolectados a diciembre de 2011. Para el análisis, se consideraron todos los registros de las personas censadas por la Secretaría de Salud y Protección Social del Distrito. 
Se empleó una matriz electrónica en Excel, que contenía las variables pertinentes para el estudio, la cual facilitó el cumplimiento del objetivo del estudio. Además, el proyecto contó con la autorización de la Secretaría de Salud de Barranquilla para la utilización de la base de datos, la cual pertenece al Ministerio de la Protección Social.

Según los parámetros de la declaración de Helsinki y la resolución 08430 de 1993 del Ministerio de Salud, este trabajo se clasifica como una investigación sin riesgo, en razón de que no se hizo manipulación de los participantes en el estudio, sino que se contó con la información de la base de datos utilizada por el Ministerio de Protección Social, a partir del registro de caracterización y localización de las personas con discapacidad (17).

Para el análisis de los datos, se utilizó el programa estadístico SPSS versión 19, con licencia adquirida por la Universidad Autónoma de Manizales. Y para la descripción del comportamiento de las variables incluidas en el estudio, se realizó un análisis univariado, a través de frecuencias absolutas y relativas. El análisis bivariado se realizó mediante tablas de contingencia, y cruzando las variables clasificadas como dependientes (deficiencia, limitación en la actividad y restricción en la participación) con los determinantes sociales de la salud (considerados como independientes). Se utilizó la prueba de Chi2 teniendo en cuenta que todas las variables se clasifican como categóricas. También se analizaron los valores de significancia bilateral para determinar la asociación entre variables, y se estableció el coeficiente de contingencia, que corrobora dicha asociación a partir de niveles de dependencia.

\section{Resultados}

En la Tabla 1, se evidencia que el mayor porcentaje de los registros (54,8\%) corresponde al género masculino. Por otra parte, el $42 \%$ de los participantes no tiene estudios; el $23 \%$ ha estudiado primaria; otro $23 \%$, secundaria; y el $1,2 \%$ ha alcanzado nivel de postgrado. Además, el $80 \%$ corresponde a estratos 1 y 2 , mientras que un $60,1 \%$ vive en la cabecera de la ciudad y el 3,4\% son rurales dispersos. En cuanto a la edad, el promedio fue de $38 \pm 19,6$ años.

Tabla 1.Distribución de determinantes estructurales socio-demográficos

\begin{tabular}{|c|c|c|c|}
\hline Variable & Indicador & Frec. (n) & Porc. (\%) \\
\hline \multirow{2}{*}{ Género } & Masculino & 398 & 54,8 \\
\hline & Femenino & 328 & 45,2 \\
\hline \multirow{21}{*}{ Edad } & $0-4$ años & 27 & 3,7 \\
\hline & 5 - 9 años & 39 & 5,4 \\
\hline & 10 - 14 años & 60 & 8,3 \\
\hline & 15 - 19 años & 62 & 8,5 \\
\hline & $20-24$ años & 52 & 7,2 \\
\hline & $25-29$ años & 55 & 7,6 \\
\hline & $30-34$ años & 61 & 8,4 \\
\hline & 35 - 39 años & 46 & 6,3 \\
\hline & 40 - 44 años & 53 & 7,3 \\
\hline & 45 - 49 años & 48 & 6,6 \\
\hline & 50 - 54 años & 41 & 5,6 \\
\hline & 55 - 59 años & 41 & 5,6 \\
\hline & 60 - 64 años & 31 & 4,3 \\
\hline & 65 - 69 años & 21 & 2,9 \\
\hline & 70 - 74 años & 24 & 3,3 \\
\hline & 75 - 79 años & 21 & 2,9 \\
\hline & 80 - 84 años & 20 & 2,8 \\
\hline & 85 - 89 años & 14 & 1,9 \\
\hline & $90-94$ años & 7 & 1,0 \\
\hline & 95 - 99 años & 2 & 0,3 \\
\hline & 100 - 105 años & 1 & 0,1 \\
\hline \multirow{7}{*}{$\begin{array}{l}\text { Nivel } \\
\text { educativo }\end{array}$} & Ninguno & 305 & 42 \\
\hline & Preescolar & 48 & 6,6 \\
\hline & Primaria & 167 & 23 \\
\hline & \multirow{4}{*}{$\begin{array}{l}\text { Secundaria } \\
\text { Técnico, } \\
\text { tecnológico } \\
\text { Universitario } \\
\text { Postgrado }\end{array}$} & 142 & 19,6 \\
\hline & & 35 & 4,8 \\
\hline & & 20 & 2,8 \\
\hline & & 9 & 1,2 \\
\hline \multirow{5}{*}{$\begin{array}{l}\text { Estrato } \\
\text { socio- } \\
\text { económico }\end{array}$} & Estrato 1 & 503 & 69,3 \\
\hline & Estrato 2 & 78 & 10,7 \\
\hline & Estrato 3 & 136 & 18,7 \\
\hline & Estrato 4 & 6 & 0,8 \\
\hline & Estrato 5 & 3 & 0,4 \\
\hline \multirow{4}{*}{ Área } & Cabecera & 436 & 60,1 \\
\hline & Centro poblado & 256 & 35,3 \\
\hline & Rural disperso & 25 & 3,4 \\
\hline & No sabe & 9 & 1,2 \\
\hline
\end{tabular}

Fuente: Datos del estudio.

Al relacionar el género con los determinantes estructurales, se encontró asociación estadísticamente significativa con la raza. Sin embargo, la dependencia entre estas variables es baja (Tabla 2).

Cienc. innov. salud. Diciembre 2014; 2 (2):63 - 69. Universidad Simón Bolívar (Col).ISSN: 2344-8636 http://portal.unisimonbolivar.edu.co:82/rdigital/innovacionsalud 
Tabla 2. Comparativo entre el género y los determinantes estructurales

\begin{tabular}{lccc}
\hline \multicolumn{1}{c}{$\begin{array}{c}\text { Género y } \\
\text { determinantes }\end{array}$} & x2 & p valor & $\begin{array}{c}\text { Coeficiente de } \\
\text { contingencia }\end{array}$ \\
\hline $\begin{array}{l}\text { Género vs. } \\
\text { Estrato }\end{array}$ & 1,54 & 0,46 & 0,046 \\
$\begin{array}{l}\text { Género vs. Nivel } \\
\text { educativo }\end{array}$ & 1,91 & 0,59 & 0,05 \\
$\begin{array}{l}\text { Género vs. Raza } \\
\text { Género vs Edad } \\
\text { (Ciclo vital) }\end{array}$ & 10,09 & $0,03 *$ & 0,11 \\
\hline
\end{tabular}

${ }^{*} \mathrm{p} \leq 0,05$, Fuente: Datos del Estudio

La Tabla 3 indica que el género presenta asociación estadísticamente significativa con las variables: tipo de afiliación al sistema de seguridad social, trabajo que desempeña y salario mensual. De igual forma, se percibe que la dependencia entre estas variables, a partir del coeficiente de contingencia, es débil.

Tabla 3. Comparativo entre el género y los determinantes intermedios

\begin{tabular}{|c|c|c|c|}
\hline $\begin{array}{l}\text { Género y } \\
\text { determinantes } \\
\text { intermedios }\end{array}$ & $\mathrm{x} 2$ & $p$ valor & $\begin{array}{l}\text { Coeficiente de } \\
\text { contingencia }\end{array}$ \\
\hline $\begin{array}{l}\text { Género vs. } \\
\text { Afiliación a salud }\end{array}$ & 3,67 & 0,159 & 0,07 \\
\hline $\begin{array}{l}\text { Género vs. Tipo de } \\
\text { afiliación }\end{array}$ & 15,46 & $0,00 *$ & 0,01 \\
\hline $\begin{array}{l}\text { Género vs. Trabajo } \\
\text { que desempeña }\end{array}$ & 8,21 & $0,04^{*}$ & 0,106 \\
\hline $\begin{array}{l}\text { Género vs. Salario } \\
\text { mensual }\end{array}$ & 12,17 & $0,002 *$ & 0,128 \\
\hline $\begin{array}{l}\text { Género vs. Tipo de } \\
\text { vivienda }\end{array}$ & 2,44 & 0,48 & 0,058 \\
\hline $\begin{array}{l}\text { Género vs. } \\
\text { Condición de } \\
\text { vivienda }\end{array}$ & 4,51 & 0,211 & 0,07 \\
\hline
\end{tabular}

${ }^{*} \mathrm{p} \leq 0,05$, Fuente: Datos del Estudio

En cuanto al género y los componentes de la discapacidad, resulta claro que no existen asociaciones estadísticamente significativas (Tabla 4).
Tabla 4. Comparativo entre el género y los componentes de la discapacidad

\begin{tabular}{llll}
\hline $\begin{array}{l}\text { Género y } \\
\text { componentes de } \\
\text { discapacidad }\end{array}$ & $\mathbf{x 2}$ & $\mathbf{p}$ valor & $\begin{array}{l}\text { Coeficiente de } \\
\text { contingencia }\end{array}$ \\
\hline $\begin{array}{l}\text { Género vs. } \\
\text { Limitaciones }\end{array}$ & 0,000 & 0,998 & 0,998 \\
$\begin{array}{l}\text { Género vs. } \\
\text { Restricción en la } \\
\text { participación }\end{array}$ & 1,155 & 0,295 & 0,283 \\
\hline$*$ p $\leq 0,05$, Fuente: Datos del Estudio & & \\
\end{tabular}

${ }^{*} \mathrm{p} \leq 0,05$, Fuente: Datos del Estudio

\section{Discusión}

En cuanto a los determinantes de la salud estructurales, las diferencias encontradas por género concuerdan con los resultados del Censo 2005 (7) para Barranquilla, el cual reporta que en el total de la población con discapacidad, la relación hombre- mujer es similar. Esto no coincide con lo establecido en España, donde la categoría femenino se ve más afectada en cuanto a condiciones de salud, al igual que quienes carecen de un ingreso mensual (18).

Para Barranquilla, además, el grupo de 10-34 años de edad es el de mayor porcentaje, representado en un $40 \%$, dato diferente al arrojado en un estudio realizado en Chile, que señala un porcentaje de $35,1 \%$ en los mayores de 60 años (16).

El estado de salud está determinado por la edad, el ingreso, el nivel educativo, la actividad laboral, la ubicación geográfica, el género, los hábitos de las personas, y la seguridad social en salud. Pero el mayor efecto marginal corresponde a la educación, lo cual implica que a medida que aumenta el nivel educativo de los individuos, también aumenta la probabilidad de contar con un buen estado de salud (19).

En el análisis del género frente a los componentes de la discapacidad, no se encontró asociación significativa, dado que tanto la categoría hombres como la categoría mujeres pueden presentar limitaciones en la actividad. Sin embargo, el 54.8\% de los hombres presenta limitaciones en la actividad, hecho contrario a lo que se evidenció en el estudio de la discapacidad de Chile antes mencionado, en el cual se registró que las mujeres tienen un mayor porcentaje de limitaciones en las actividades (16).

La raza, como factor personal $(20,21)$ se clasifica entre las circunstancias de construcción social y es un 
factor de exclusión que influye en las desigualdades de salud, así como el género.

De acuerdo con la CIF, (7), las personas que presentan alguna deficiencia no necesariamente tienen alguna limitación o restricción en la participación. De ahí la importancia de caracterizar a cada individuo en términos de funcionamiento para así establecer un plan de rehabilitación o habilitación. Con otras palabras: "los niveles de funcionamiento varían enormemente dentro de una población, ya sea en relación con las capacidades físicas, intelectuales, habilidades sensoriales, o como resultado del impacto de la salud mental. Si estas diferencias no se toman en cuenta se puede limitar seriamente la efectividad de los programas diseñados para promover el bienestar económico y social"(22). Esto último también es coherente con lo planteado por la CIF, según la cual las deficiencias están relacionadas con la discapacidad, pero no son determinantes de la misma, es decir, que tener deficiencias no implica poseer una discapacidad (7).

De acuerdo con la CIF, la expresión "Discapacitados sociales", acuñada para destacar la dificultad de participación que tienen ciertas personas debido a condiciones socioculturales, también se utiliza en los casos en que circunstancias como pobreza y violencia producen problemas de salud que llevan a la discapacidad (23). Similares resultados se encontraron en el presente estudio, pues en este se muestra que una patología especifica también se ve fuertemente influenciada por los determinantes sociales como : área de residencia, afiliación y acceso a los servicios de salud, estrato socioeconómico, los cuales son factores determinantes de la presencia o no de la enfermedad. En cambio, los determinantes sociales como género, condiciones de vivienda o asistencia a programas de crecimiento y desarrollo no están asociados significativamente a la presencia de enfermedad (22).

Según la CELAP (24), en la gran mayoría de los países, las tasas de discapacidad de los hombres supera a las de las mujeres desde su nacimiento hasta los 40 años, situación que cambia en el siguiente ciclo de vida. Así, al llegar al grupo de 60 años y más, la prevalencia de las mujeres sobrepasa la de los hombres en todos los países del Caribe y en 11 de los 17 países de América Latina de los que se tienen datos. De manera similar, en los resultados de la ciudad de Barranquilla, se encontró que la discapacidad se presenta en mayores de 60 años y con mayor frecuencia en mujeres. Este cambio de patrón con relación a la edad entre hombres y mujeres, se podría explicar por el hecho de que la mayor esperanza de vida de las mujeres aumenta la posibilidad de tener una discapacidad generada por un accidente o una enfermedad crónica (24).

Se concluye que los registros en estudio presentaron porcentajes similares en cuanto a género. El promedio

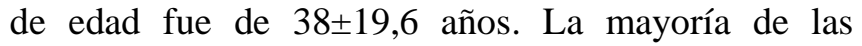
personas no tiene ningún nivel educativo, son de raza mestiza y vive en la cabecera, además de pertenecer al estrato 1 y 3 . Por último, solo cerca del $19 \%$ recibe algún ingreso económico mensual.

Se puede afirmar, por tanto, que existe asociación estadísticamente significativa entre el género y la raza como determinante estructural. En cuanto al género y los determinantes intermedios, los que presentaron asociaciones estadísticas fueron: trabajo que desempeña, salario mensual y tipo de afiliación. No se evidenció asociación entre el género y los componentes de la discapacidad.

Finalmente, la discapacidad resulta evidente cuando la persona encuentra o presenta restricciones que le impiden su plena participación en la sociedad. Las personas con limitaciones físicas tienen discapacidades no tanto en cuanto a su nivel de accionar sino porque se les niega el acceso a la educación, mercado laboral y servicios públicos y de salud. De manera que la discapacidad no se encuentra en el individuo, sino más bien en las condiciones bio psicosociales que lo determinan.

Conflicto de Intereses: Los autores declaran que no se presentaron conflictos de interés en el desarrollo de este estudio.

\section{Referencias}

1. Tovar LM, García G. El entorno regional y la percepción del estado de salud en Colombia. Lect. Econ. 2003; 65:177-208.

2. Álvarez LS. Los determinantes sociales de la salud: Más allá de los factores de riesgo. Rev. Gerenc. Polit. Salud. 2009; 8 (17): 69-79.

3. Dahlgren G, Whitehead M. Policies and strategiestopromote social equity in healthStockholm. Stockholm: Institute of 
FuturesStudies; 1991. Disponible en www.who.int/bulletin/volumes/84/.../index.html-

4. Comisión de los Determinantes de Salud. Lograr la equidad en salud: desde las causas iniciales a los resultados justos. Declaración provisional. Organización Mundial de la Salud. Ginebra; 2007.

5. PublicHealth Agency of Canada. The Social Determinants of Health: anoverview of theimplicationsforpolicy and the role of thehealth sector. Ottawa; 2003.

6. Organización Mundial de la Salud. Clasificación Internacional del Funcionamiento, de la Salud y la Discapacidad (CIF). Madrid; 2001.

7. Departamento Administrativo Nacional de Estadística (DANE). Dirección de censos y demografía. Base de datos de registro de localización y caracterización de personas con discapacidad. Bogotá; 2005.

8. Commissionon Social Determinants of Health. A conceptual frameworkforactiononthe social determinants of health. Geneva: WorldHealthOrganization; 2007.

9. Cruz I, Hernández J. Magnitud de la discapacidad en Colombia: una aproximación a sus determinantes. Rev. Cienc. Salud. 2008; 6 (3): 23-35.

10. Saldarriaga Concha. La discapacidad en el contexto del Sistema General de Seguridad Social en Salud en Colombia: Lineamientos, epidemiología e impacto económico. In: Fundación Santa Fe de Bogotá, editor. Bogotá D.C; 2009. Pp.1-7.

11. Consejo Nacional de Política Económica y Social. Documento Conpes Social 80. Política Pública Nacional de Discapacidad. Bogotá, 2004.

12. Ospina RS. Programa de Discapacidad Red de Solidaridad Social. Estudio de prevalencia de Discapacidad. Revisión de estudios sobre situación de discapacidad en Colombia. Cali, Valle del Cauca, 2001.

13. Zitko P, Araya R. Cabieses B. Determinantes Socioeconómicos de Discapacidad. RevChil Salud Pública. 2010.

14. Instituto Universitario de Estudios Europeos. La accesibilidad en España, diagnóstico y bases para un plan integral de supresión de barreras. IMSERSO Universidad Autónoma de Barcelona, 2002. p. 341.
15. Fondo Nacional de la Discapacidad (FONADIS). Primer estudio de la discapacidad en Chile. 2005.

16. Organización Mundial de la Salud. Informe mundial sobre la violencia y la salud. Washington DC; 2002.

17. Ministerio de salud. Resolución 8430 de 1993. Normas científicas, técnicas y administrativas para la investigación en salud. Bogotá; 1993.

18. Borrell C, Rodríguez M, Pérez G, García AA. Las desigualdades sociales en salud en el Estado español. Aten Primaria. 2008; 40 (2):59-60.

19. Tovar L. Determinantes del estado de salud de la población colombiana. Econ. Gest. Desarro. 2005; 3: 125-151.

20. Weber LA. Conceptual frameworkforunderstandingrace, class, gender and sexuality. Psychology of WomenQuarterly. 1998; 22:13-32.

21. Weber LA. Reconstructingthelandscape of healthdisparitiesresearch. Promoting dialogue and collaborationbetweenfeministintersectional and biomedicalparadigms. In: Schulz AJ, Mullings L, editores. Gender, race, class, and health. Intersectionalapproaches. San Francisco: JosseyBass; 2006, pp. 21-59.

22. Astorga LF. Incluyendo a las personas con discapacidad en las políticas de desarrollo. Managua, Nicaragua, 2007. p. 3.

23. Arrieta R, Caro G. Determinantes sociales y disparidades de la morbilidad por enfermedades respiratorias agudas (ERA) en niñas y niños de uno a cuatro años en Colombia. Rev. Gerenc. Polit. Salud. 2010; 9 (18): 137-156.

24. Comisión Económica para América Latina y el Caribe ECLA (Ingles) - CELAP. Autonomía e independencia: El cuidado de las personas con discapacidad. Versión preliminar. 2012. 